Audiology

Neurotology
Audiol Neurotol 2018;23:259-269

DOI: $10.1159 / 000494049$
Received: February 9, 2018

Accepted after revision: September 25, 2018

Published online: December 11, 2018

\title{
Comparison of Pure-Tone Average Methods for Estimation of Hearing Loss Caused by Environmental Exposure to Lead and Cadmium: Does the Pure-Tone Average Method Which Uses Low-Frequency Ranges Underestimate the Actual Hearing Loss Caused by Environmental Lead and Cadmium Exposure?
}

\author{
Da-An Huh ${ }^{a}$ Yun-Hee Choi ${ }^{b}$ Myung Sun Jic Kyong Whan Moon ${ }^{c}$ Seok J. Yoon ${ }^{d}$ \\ Jong-Ryeul Sohn ${ }^{d}$ \\ ${ }^{a}$ Department of Public Health Sciences, Graduate School, Korea University, Seoul, Republic of Korea; ${ }^{b}$ Department of \\ Health and Safety Convergence Science, Graduate School, Korea University, Seoul, Republic of Korea; ' ${ }^{\circ}$ Department of \\ Health and Environmental Science, College of Health Science, Korea University, Seoul, Republic of Korea; ${ }^{\text {BK}} 21$ PLUS \\ Program in Embodiment: Health-Society Interaction, Department of Public Health Sciences, Graduate School, Korea \\ University, Seoul, Republic of Korea
}

\section{Keywords}

Cadmium · Hearing $\cdot$ Lead $\cdot$ Pure-tone average

\begin{abstract}
Previous studies have reported that exposure to lead and cadmium can damage the inner ear receptor, which perceives high-frequency sounds. However, few studies have used the pure-tone average (PTA), including high-frequency ranges, for the estimation of hearing loss caused by lead and cadmium exposure. We estimated hearing loss using the PTA test, in low-frequency, speech frequency, and high-frequency ranges and compared the differences in the results using 3 PTA calculation methods. We analyzed the data of 2,387 participants, between the ages of 19 and 85 years, that were obtained from the Korea National Health and Nutrition Examination Survey (KNHANES) of 2010-2012. A dose-response relationship between hearing loss and heavy metal exposure was observed in the high-frequency method after
\end{abstract}

adjustment for confounding factors. When using the highfrequency PTA, it was found that doubling of the levels of lead and cadmium in the blood was associated with a 1.88(95\% Cl 1.11-3.17) and 1.89-fold (95\% Cl 1.02-3.50) increase in the OR for hearing loss. In the case of the low-frequency and speech frequency PTA, however, there were no significant relationships between hearing loss and the concentrations of lead and cadmium in the blood. The outcomes of the present study suggest that the estimation of hearing loss caused by environmental exposure to lead and cadmium is affected by the frequencies used in the PTA calculation.

(C) 2018 The Author(s)

Published by S. Karger AG, Basel

\section{Introduction}

Previous studies have suggested that environmental exposure to cadmium and lead, both ototoxic chemicals, is associated with hearing loss. Jones et al. [2008] reported

\begin{tabular}{ll}
\hline KARGER & $\begin{array}{l}\text { (๑) } 2018 \text { The Author(s) } \\
\text { Published by S. Karger AG, Basel }\end{array}$ \\
E-Mail karger@karger.com & $\begin{array}{l}\text { This article is licensed under the Creative Commons Attribution- } \\
\text { NonCommercial-NoDerivatives 4.0 International License (CC BY- } \\
\text { NC-ND) (http://www.karger.com/Services/OpenAccessLicense). } \\
\text { Usage and distribution for commercial purposes as well as any dis- } \\
\text { tribution of modified material requires written permission. }\end{array}$
\end{tabular}


that lead exposure induces degeneration of the inner ear receptor cells and decreases the velocity of conduction in the auditory nerves. Kim et al. [2008] presented evidence of cadmium exposure inducing apoptosis and reversing the receptor cell arrangement of the inner ear, causing an increment of the hearing threshold. The more basal region of the cochlea is more susceptible to ototoxicity, and the inner ear receptor perceives high-frequency sounds [Musiek, 2006; Liu et al., 2014]; therefore, people exposed to lead or cadmium could have high-frequency hearing loss.

The pure-tone average (PTA) is the most commonly used assessment tool for estimation of hearing loss. The hearing ability of a person can be easily determined using the average of hearing threshold levels at specified frequencies; however, there is no international guideline from which to choose the appropriate frequencies. A few studies that reported the association between heavy metal exposure and hearing loss calculated the PTA variably in the frequency range of $0.5-8 \mathrm{kHz}$, and most of those studies commonly used the range $0.5-4 \mathrm{kHz}$ (the socalled speech frequency) [Counter et al., 1997; Chuang et al., 2007; Hwang et al., 2009; Park et al., 2010; Shargorodsky et al., 2011; Choi et al., 2012; Saunders et al., 2013; Choi and Kim, 2014; Huh et al., 2016; Choi and Park, 2017]. However, the PTA that is determined using the speech frequency is likely to underestimate the degree of hearing loss, because lead and cadmium affect the highfrequency hearing ability rather than the low-frequency hearing ability.

In the present study, therefore, we statistically estimated the frequency of hearing that is most affected by increased lead and cadmium exposure levels, in the Korean population. We also evaluated hearing loss using the PTA in the low frequency, speech frequency, and high frequency, and compared the differences between the results, using 3 PTA calculation methods.

\section{Materials and Methods}

\section{Study Population}

The Korea National Health and Nutrition Examination Survey (KNHANES), which is conducted by the Korea Centers for Disease Control and Prevention, is a nationwide survey that represents the general South Korean population. KNHANES is an ongoing series of cross-sectional surveys that aims to obtain health-related information such as health and nutrition statuses, as well as the lifestyle and sociodemographic characteristics of the population. The dataset is provided periodically by the KNHANES website (https:// knhanes.cdc.go.kr), and it is available to all researchers.

As part of the KNHANES, in 2010-2013, audiometric examinations were conducted. In addition, information on blood metal measurements and noise exposure statuses, which are important confounders, was available from 2010-2012; therefore, the present analysis used data from the KNHANES 2010-2012. We combined the data of 25,534 participants from these 3 years and excluded 23,147 participants on whom audiometric or heavy metal examinations, as well as interviews regarding covariates (monthly income, education level, smoking status, height, weight, noise exposure status, hypertension, and diabetes mellitus), were not conducted. As a result of exclusion, we selected 2,387 participants who had the complete dataset as described above.

Blood Lead and Cadmium Levels

The lead $(\mathrm{Pb})$ and cadmium $(\mathrm{Cd})$ levels in the blood samples were measured using graphite furnace atomic absorption spectrometry (model AAnalyst 600; PerkinElmer, Finland). For internal quality assurance, the analytical equipment was controlled using standard reference material from Whole Blood Metals Control (Bio-Rad, USA). External quality assurance was satisfied by the German External Quality Assessment (G-EQUAS). The limit of detection was $0.12 \mu \mathrm{g} / \mathrm{dL}$ for blood lead and $0.056 \mu \mathrm{g} / \mathrm{L}$ for blood cadmium, based on data from the KNHANES (2010-2012). None of the samples exhibited values below the limit of detection, for either lead or cadmium.

\section{Audiometric Measurement}

The audiometric measurement was performed in a sound-isolated booth inside a mobile examination center. Resident physicians, experts in otorhinolaryngology, performed all of the audiometric testing. The pure-tone audiometric examination was performed using an audiometer (model SA203; LenaNodin, Sweden), according to the procedure defined by the Korean Society of Otorhinolaryngology-Head and Neck Surgery. The test frequencies of the hearing thresholds were $0.5,1,2,3,4$, and $6 \mathrm{kHz}$, with an intensity range of -10 to $110 \mathrm{~dB}$. We computed the PTA of hearing thresholds at low frequencies $(0.5,1$, and $2 \mathrm{kHz})$, speech frequencies $(0.5,1,2$, and $4 \mathrm{kHz})$ and high frequencies $(3,4$, and $6 \mathrm{kHz})$ [Agrawal et al., 2008]. Hearing loss was defined as a PTA $>25 \mathrm{~dB}$, in either ear, according to the definition put forth by the World Health Organization [1991].

\section{Covariates}

We used demographic and hearing-related variables as potential confounders. The variables that we considered were age, sex, monthly income, education level, smoking status, BMI, noise exposure status (occupational noise, loud noise exposure, and firearm noise exposure), hypertension, and diabetes mellitus. Data on monthly incomes were available as quartiles each survey year, and education level was classified as middle school or less (reference), high school graduation, or college or more. Smoking status was categorized as nonsmoker (reference), past smoker, or current smoker. The BMI was calculated as weight $(\mathrm{kg}) /$ height $(\mathrm{m})$ squared. Based on the self-reported exposure to occupational noises, loud noises, and firearm noises, participants were classified into the nonexposed group (reference) and the exposed group. Participants were assigned to the "exposed group" in terms of occupational noise if they reported exposure to noise in the workplace for over 3 months. The exposed group, in relation to exposure to loud noises, consisted of those who reported exposure to loud noise (loud music, loud car horns, and loud machinery) outside of the workplace for more than $5 \mathrm{~h}$ a week. Exposure to firearm noise was 
Table 1. Age-adjusted geometric means of variables by participant characteristic

\begin{tabular}{|c|c|c|c|c|}
\hline \multirow[b]{2}{*}{ Variable } & \multicolumn{2}{|c|}{ Blood lead $(n=2,387)$} & \multicolumn{2}{|c|}{ Blood cadmium $(n=2,387)$} \\
\hline & $\mu \mathrm{g} / \mathrm{dL}(95 \% \mathrm{CI})^{\mathrm{a}}$ & $p$ value $^{\mathrm{b}}$ & $\mu \mathrm{g} / \mathrm{L}(95 \% \mathrm{CI})^{\mathrm{a}}$ & $p$ value $^{\mathrm{b}}$ \\
\hline Total & $2.46(2.41-2.52)$ & & $1.02(0.99-1.05)$ & \\
\hline \multicolumn{5}{|l|}{ Age (years) } \\
\hline $19-39$ & $2.07(2.02-2.13)$ & $<0.001$ & $0.85(0.81-0.89)$ & $<0.001$ \\
\hline $40-59$ & $2.72(2.64-2.80)$ & & $1.13(1.09-1.18)$ & \\
\hline$\geq 60$ & $2.90(2.74-3.06)$ & & $1.20(1.13-1.28)$ & \\
\hline \multicolumn{5}{|l|}{ Sex } \\
\hline Male & $2.56(2.52-2.61)$ & $<0.001$ & $1.00(0.97-1.03)$ & $<0.001$ \\
\hline Female & $1.93(1.82-2.05)$ & & $1.13(1.05-1.22)$ & \\
\hline \multicolumn{5}{|l|}{ Monthly income } \\
\hline Low & $2.37(2.24-2.50)$ & 0.195 & $1.11(1.02-1.20)$ & 0.129 \\
\hline Low-middle & $2.52(2.44-2.60)$ & & $1.01(0.96-1.06)$ & \\
\hline High-middle & $2.44(2.35-2.53)$ & & $0.99(0.94-1.04)$ & \\
\hline High & $2.49(2.40-2.58)$ & & $1.01(0.97-1.06)$ & \\
\hline \multicolumn{5}{|l|}{ Education level } \\
\hline$<$ High school & $2.49(2.37-2.63)$ & $<0.001$ & $1.07(1.00-1.14)$ & $<0.001$ \\
\hline High school & $2.58(2.49-2.67)$ & & $1.11(1.06-1.15)$ & \\
\hline$>$ High school & $2.36(2.30-2.42)$ & & $0.93(0.89-0.97)$ & \\
\hline \multicolumn{5}{|l|}{ Smoking status } \\
\hline Nonsmoker & $2.28(2.22-2.35)$ & $<0.001$ & $0.81(0.78-0.84)$ & $<0.001$ \\
\hline Past smoker & $2.30(2.11-2.50)$ & & $0.88(0.78-1.00)$ & \\
\hline Current smoker & $2.65(2.58-2.73)$ & & $1.27(1.23-1.31)$ & \\
\hline \multicolumn{5}{|l|}{ BMI } \\
\hline$<25$ & $2.42(2.36-2.49)$ & 0.012 & $1.01(0.98-1.05)$ & 0.509 \\
\hline $25-30$ & $2.56(2.49-2.63)$ & & $1.02(0.98-1.06)$ & \\
\hline$\geq 30$ & $2.44(2.28-2.60)$ & & $1.10(0.96-1.25)$ & \\
\hline \multicolumn{5}{|l|}{ Occupational noise exposure } \\
\hline No & $2.41(2.36-2.47)$ & $<0.001$ & $1.00(0.97-1.03)$ & 0.003 \\
\hline Yes & $2.70(2.59-2.82)$ & & $1.11(1.04-1.17)$ & \\
\hline \multicolumn{5}{|l|}{ Loud noise exposure } \\
\hline No & $2.46(2.41-2.51)$ & 0.451 & $1.02(0.99-1.05)$ & 0.869 \\
\hline Yes & $2.59(2.27-2.94)$ & & $1.03(0.86-1.24)$ & \\
\hline \multicolumn{5}{|l|}{ Firearm noise exposure } \\
\hline No & $2.42(2.36-2.49)$ & 0.026 & $1.02(0.98-1.05)$ & 0.806 \\
\hline Yes & $2.53(2.46-2.60)$ & & $1.02(0.98-1.07)$ & \\
\hline \multicolumn{5}{|c|}{ Current diagnosis of hypertension } \\
\hline Normal & $2.37(2.29-2.44)$ & 0.002 & $0.99(0.95-1.03)$ & 0.095 \\
\hline Prehypertension & $2.55(2.46-2.64)$ & & $1.02(0.97-1.07)$ & \\
\hline Hypertension & $2.55(2.46-2.65)$ & & $1.07(1.01-1.12)$ & \\
\hline \multicolumn{5}{|l|}{ Current diagnosis of diabetes } \\
\hline Normal & $2.45(2.39-2.51)$ & 0.101 & $1.01(0.98-1.04)$ & 0.268 \\
\hline Impaired fasting glucose & $2.56(2.46-2.67)$ & & $1.06(1.01-1.12)$ & \\
\hline Diabetes & $2.37(2.21-2.55)$ & & $0.99(0.91-1.09)$ & \\
\hline
\end{tabular}

${ }^{\text {a }}$ Age-adjusted value, except for age groups. ${ }^{\mathrm{b}} t$ test or Wald $F$ test.

defined as exposure to instantaneous loud explosions or gunfire sounds. Hypertension was categorized into the following 3 groups: systolic blood pressure $<120 \mathrm{~mm} \mathrm{Hg}$ and diastolic blood pressure $<80 \mathrm{~mm} \mathrm{Hg}$, normal; $120 \mathrm{~mm} \mathrm{Hg} \leq$ systolic blood pressure < 140 $\mathrm{mm} \mathrm{Hg}$ or $80 \mathrm{~mm} \mathrm{Hg} \leq$ diastolic blood pressure $<90 \mathrm{~mm} \mathrm{Hg}$, prehypertension; and systolic blood pressure $\geq 140 \mathrm{~mm} \mathrm{Hg}$ or dia- stolic blood pressure $\geq 90 \mathrm{~mm} \mathrm{Hg}$, hypertension. The presence of diabetes was classified into the following 3 groups: fasting glucose $<100 \mathrm{mg} / \mathrm{dL}$, normal; $100 \mathrm{mg} / \mathrm{dL} \leq$ fasting glucose $\leq 125 \mathrm{mg} / \mathrm{dL}$, impaired fasting glucose; and fasting glucose $>125 \mathrm{mg} / \mathrm{dL}$, diabetes. 


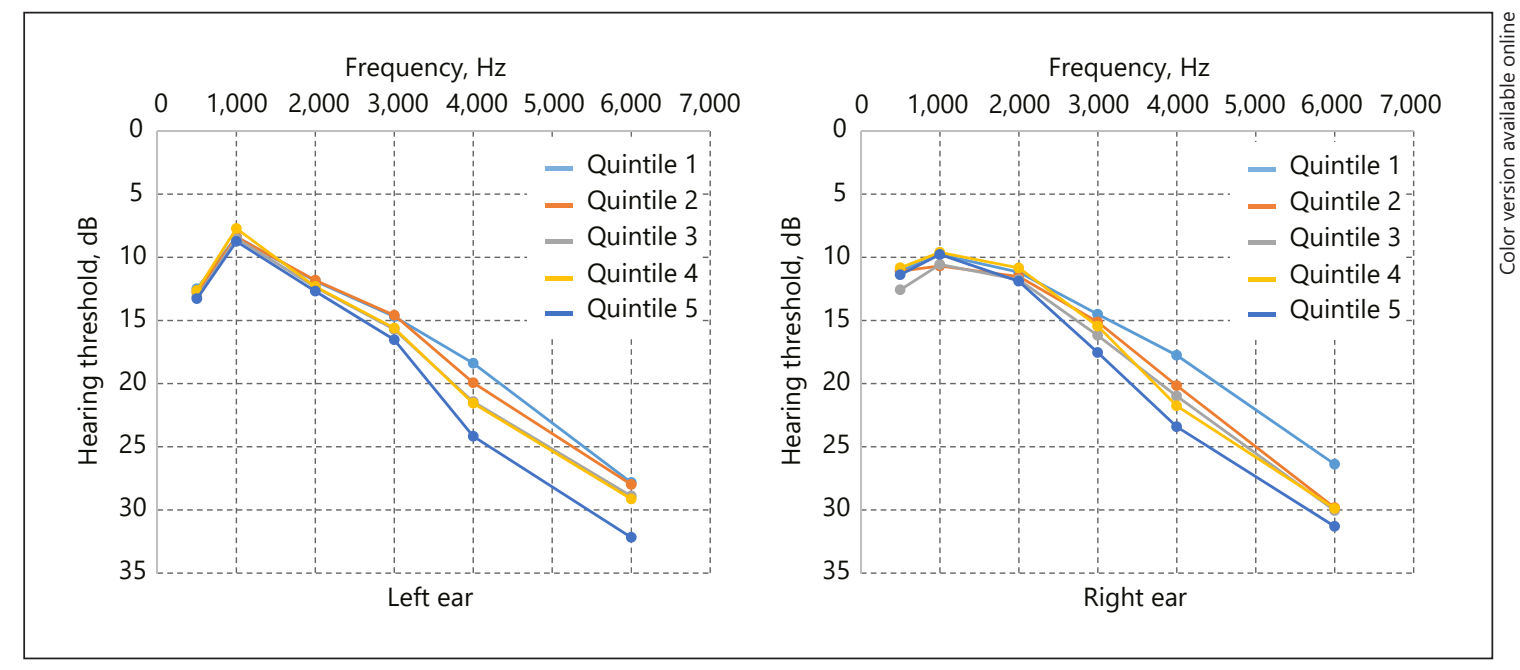

Fig. 1. Estimated hearing threshold at $0.5-6 \mathrm{kHz}$ in both ears, by lead quintile. Models were adjusted for age and noise exposure.

Statistical Analysis

The data from the KNHANES were analyzed considering the stratified multistage probability sampling design and weighted sample values were used to provide a sample of participants that was representative of the general South Korean population. For our analyses, we used an integrated weight value through the combined 2010-2012 KNHANES database, and applied it to the statistical analysis according to the KNHANES analysis tutorial [Korean Centers for Disease Control and Prevention, 2014].

We used a log transformation of blood lead and cadmium to normalize the distribution prior to the analyses. The Student $t$ test and the Wald $F$ test were used to evaluate differences in the geometric mean between the groups. ANCOVA was conducted to estimate the age- and gender-adjusted mean of hearing thresholds for each frequency.

We also used logistic regression analysis to estimate the $\mathrm{OR}$ for hearing loss. Blood lead and cadmium levels were divided into quintiles, and OR were estimated by comparing the values of each quintile with those of the lowest quintile. We constructed a single model adjusted for age, sex, monthly income, education level, smoking status, BMI, occupational noise exposure, loud noise exposure, firearm noise exposure, hypertension, and diabetes.

The joint effect of lead and cadmium exposure on hearing loss was examined after adjusting for all of the variables. We used combinations of categorical variables and classified them into the following 4 groups: low lead and low cadmium (reference), low lead and high cadmium, high lead and low cadmium, and high lead and high cadmium. The high and low categories were defined by the median values of each variable. We calculated 2 scales of interaction, an additive scale as the relative excess risk due to interaction, and a multiplicative scale as the ratio of OR, following the recommendation of Knol and VanderWeele [2012]. We also computed 95\% CI for the relative excess risk due to interaction, following the standard delta method based on a Taylor series expansion [Hosmer and Lemeshow, 1992].

We performed all statistical analyses using SPSS version 22.0, and $p<0.05$ was considered statistically significant.

\section{Results}

Table 1 presents the characteristics of the study population. The age-adjusted geometric means (95\% CI) of blood lead and cadmium levels in the whole population were 2.46 (95\% CI $2.41-2.52)$ and $1.02 \mu \mathrm{g} / \mathrm{L}$ (95\% CI $0.99-1.05)$, respectively. Blood lead and cadmium concentrations increased with age and were likely to be higher in smokers. Additionally, blood lead and cadmium levels were significantly different depending on sex, education level, and occupational noise exposure.

Figures 1 and 2 show the estimated hearing thresholds of each frequency by lead and cadmium quintiles. We adjusted for age and noise exposure to control the main effects on hearing loss. In the case of lead, the hearing thresholds of both ears did not show significant differences between quintiles at $0.5,1$, and $2 \mathrm{kHz}$, but they increased with blood lead quintiles at 3,4 , and $6 \mathrm{kHz}$ (Fig. 1). In the case of cadmium, however, there were no significant differences in hearing ability at any of the examined frequencies (Fig. 2).

We estimated the OR for hearing loss at each frequency after adjusting for age, sex, monthly income, education level, smoking status, BMI, noise exposure status, and diagnosis of hypertension and diabetes (Tables 2, 3). At 4 $\mathrm{kHz}$, the OR for hearing loss was positively associated with higher blood lead levels in the left ear (OR 1.76; 95\% CI 1.04, 2.99) and cadmium levels in the right ear (OR 1.95 ; $95 \%$ CI 1.11-3.40). 


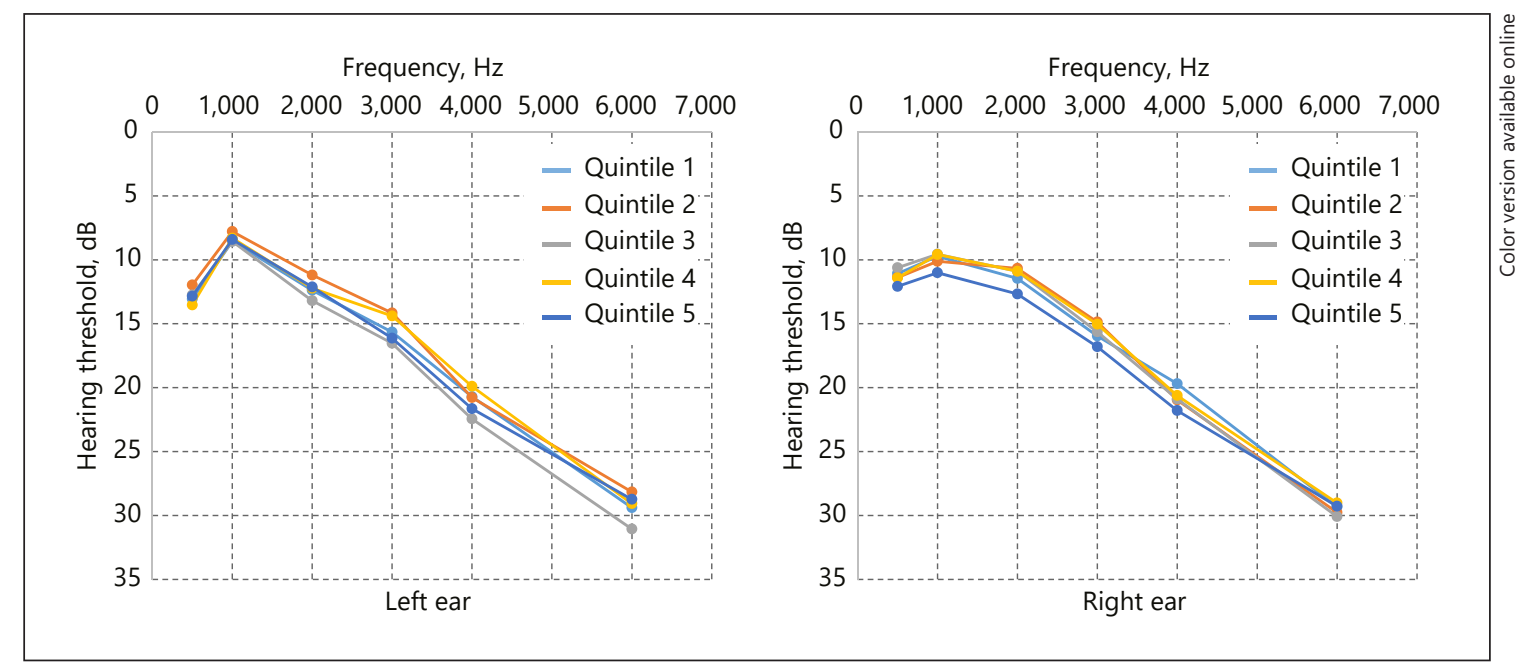

Fig. 2. Estimated hearing threshold at $0.5-6 \mathrm{kHz}$ in both ears, by cadmium quintile. Models were adjusted for age and noise exposure.

Table 2. OR for hearing loss at $0.5-6 \mathrm{kHz}$ in both ears by lead quintile

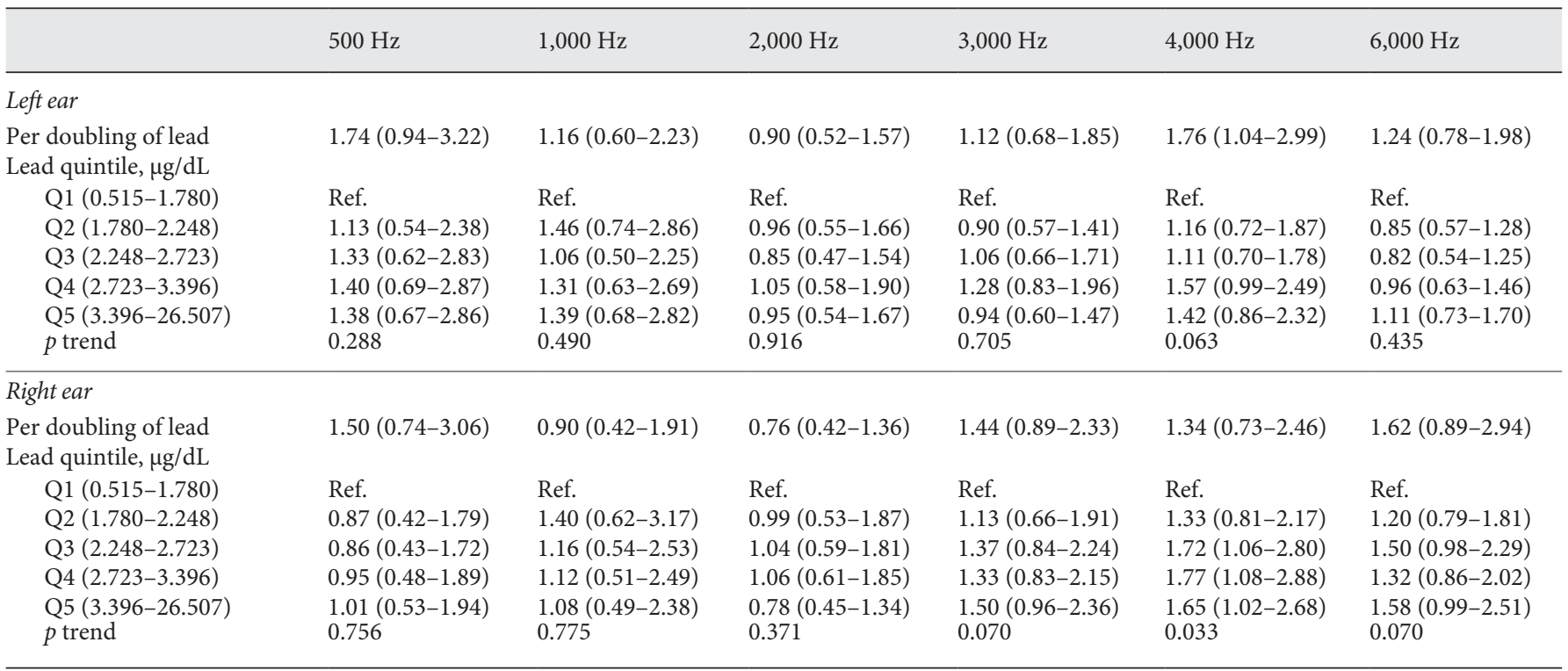

Values in parentheses are 95\% CI. Models were adjusted for age, sex, monthly income, education level, smoking status, BMI, occupational noise, loud noise, firearm noise, hypertension, and diabetes. Hearing loss: hearing threshold $>25 \mathrm{~dB}$.

Table 4 presents the result of the logistic regression for hearing loss in different PTA calculation methods. In the high-frequency method, doubling of the concentration of blood lead and cadmium was associated with a 1.88- $(95 \%$ CI 1.11-3.17) and 1.89-fold (95\% CI 1.02-3.50) increase in the OR for hearing loss. In the low-frequency and speech frequency methods, however, there were no sig- nificant associations between hearing loss and blood concentrations of lead and cadmium.

Tables 5-7 show the effects of combined exposure to lead and cadmium on hearing outcomes in the 3 PTA computing methods. In the high-frequency method, the OR for participants in both of the high groups, compared to the reference group (which had low lead and cadmium 
Table 3. OR for hearing loss at $0.5-6 \mathrm{kHz}$ in both ears by cadmium quintile

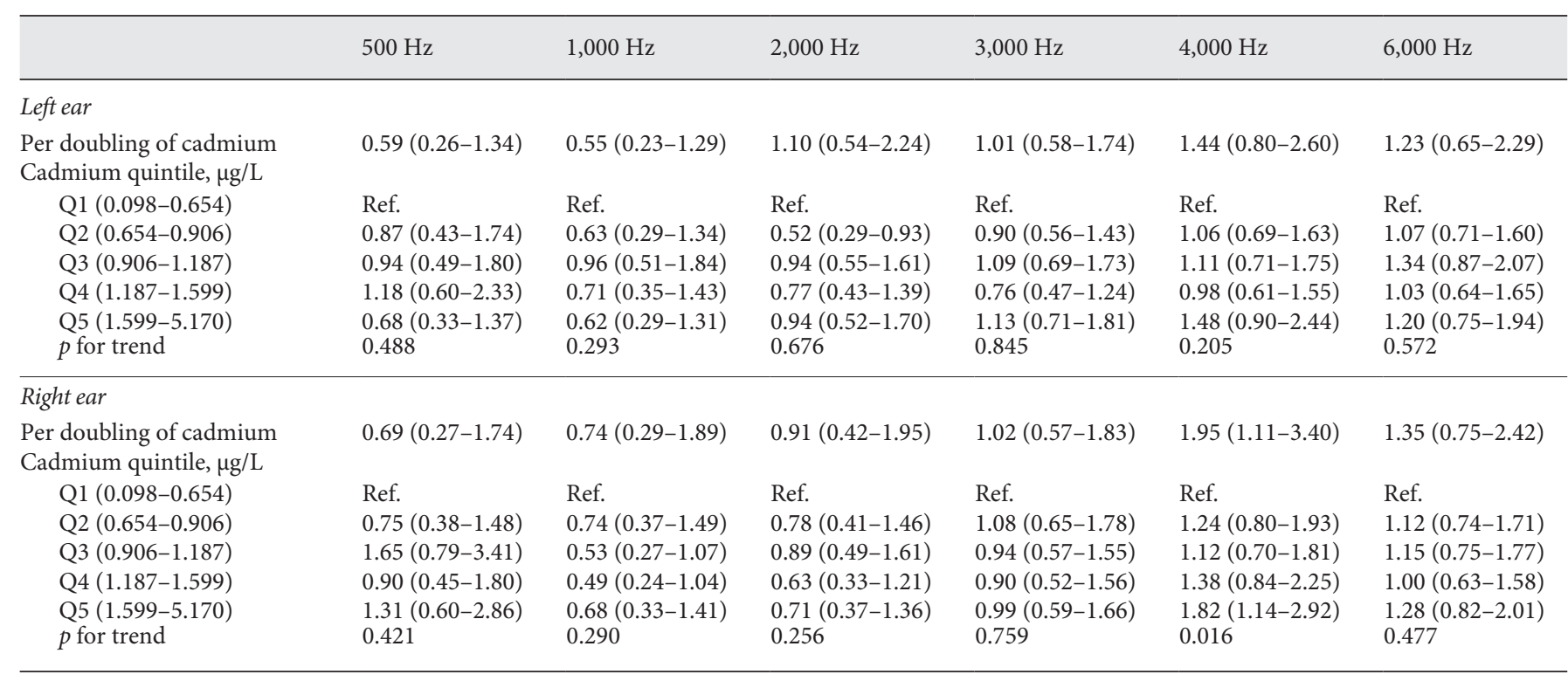

Values in parentheses are 95\% CI. Models were adjusted for age, sex, monthly income, education levels, smoking status, BMI, occupational noise, loud noise, firearm noise, hypertension, and diabetes. Hearing loss: hearing threshold $>25 \mathrm{~dB}$. Ref., reference.

Table 4. OR for hearing loss based on 3 PTA methods by lead and cadmium quintile

\begin{tabular}{|c|c|c|c|c|}
\hline Variables & $n$ & \multicolumn{3}{|l|}{ Hearing loss ${ }^{\mathrm{a}}$} \\
\hline Per doubling of lead & & $0.91(0.52-1.61)$ & $1.21(0.72-2.04)$ & $1.88(1.11-3.17)$ \\
\hline \multicolumn{5}{|l|}{ Lead quintile, $\mu \mathrm{g} / \mathrm{dL}$} \\
\hline Q1 (0.515-1.780) & 478 & Ref. & Ref. & Ref. \\
\hline Q4 (2.723-3.396) & 478 & $1.00(0.58-1.72)$ & $1.29(0.77-2.16)$ & $1.51(0.97-2.36)$ \\
\hline Q5 (3.396-26.507) & 477 & $0.86(0.50-1.48)$ & $1.27(0.77-2.11)$ & $1.44(0.90-2.31)$ \\
\hline$p$ for trend & & 0.994 & 0.673 & 0.035 \\
\hline \multicolumn{5}{|l|}{ Blood cadmium } \\
\hline Per doubling of cadmium & & $0.83(0.40-1.72)$ & $1.24(0.66-2.34)$ & $1.89(1.02-3.50)$ \\
\hline \multicolumn{5}{|l|}{ Cadmium quintile, $\mu \mathrm{g} / \mathrm{dL}$} \\
\hline Q4 (1.187-1.599) & 479 & $0.68(0.37-1.27)$ & $0.89(0.51-1.55)$ & $1.22(0.76-1.95)$ \\
\hline Q5 (1.599-5.170) & 476 & $0.71(0.37-1.38)$ & $1.29(0.72-2.32)$ & $1.72(1.05-2.83)$ \\
\hline$p$ for trend & & 0.539 & 0.502 & 0.069 \\
\hline
\end{tabular}

Values in parentheses are 95\% CI. Models were adjusted for age, sex, monthly income, education level, smoking status, BMI, occupational noise, loud noise, firearm noise, hypertension, and diabetes. ${ }^{a}$ Defined as a PTA $>25 \mathrm{~dB} .{ }^{\mathrm{b}} \mathrm{PTA}$ at $0.5,1$, and $2 \mathrm{kHz} .{ }^{\mathrm{c}} \mathrm{PTA}$ at $0.5,1,2$, and $4 \mathrm{kHz}{ }^{\mathrm{d}} \mathrm{PTA}$ at 3,4 , and $6 \mathrm{kHz}$. 
Table 5. OR for hearing loss by joint effect between blood lead and cadmium levels with the low-frequency method

\begin{tabular}{llll}
\hline Variable & Low lead & High lead & $\begin{array}{l}\text { Lead within strata } \\
\text { of cadmium }\end{array}$ \\
\hline Low cadmium & Ref. & $1.26(0.76-2.11)$ & $1.26(0.76-2.11)$ \\
High cadmium & $1.28(0.74-2.20)$ & $1.13(0.66-1.93)$ & $0.86(0.57-1.31)$ \\
Cadmium within strata of lead & $1.28(0.74-2.20)$ & $0.97(0.63-1.49)$ & \\
\hline
\end{tabular}

Values in parentheses are $95 \%$ CI. Hearing loss was defined as a PTA $>25 \mathrm{~dB}$. The low-frequency method was used with PTA at $0.5,1$, and $2 \mathrm{kHz}$. Measurement of interaction on an additive scale: RERI $=-0.41(-1.24$ to 0.41$)$; $p=0.327$. Measurement of interaction on a multiplicative scale: ratio of ORs $=0.70(0.37$ to 1.31$) ; p=0.264$. Models were adjusted for age, sex, monthly income, education level, smoking status, BMI, occupational noise, loud noise, firearm noise, hypertension, and diabetes.

Table 6. OR for hearing loss by joint effect between blood lead and cadmium levels with the speech frequency method

\begin{tabular}{llll}
\hline Variable & Low lead & High lead & $\begin{array}{l}\text { Lead within strata } \\
\text { of cadmium }\end{array}$ \\
\hline Low cadmium & Ref. & $1.28(0.83-1.97)$ & $1.28(0.83-1.97)$ \\
High cadmium & $1.33(0.82-2.15)$ & $1.11(0.74-1.66)$ & $0.77(0.49-1.20)$ \\
Cadmium within strata of lead & $1.33(0.82-2.15)$ & $0.90(0.59-1.38)$ & \\
\hline
\end{tabular}

Values parentheses are 95\% CI. Hearing loss was defined as PTA $>25 \mathrm{~dB}$. Speech frequency method: PTA at $0.5,1,2$, and $4 \mathrm{kHz}$. Measurement of interaction on an additive scale: RERI $=-0.50(-1.32$ to 0.31$) ; p=0.228$. Measurement of interaction on a multiplicative scale: ratio of ORs $=0.65(0.35$ to 1.20$) ; p=0.169$. Models were adjusted for age, sex, monthly income, education level, smoking status, BMI, occupational noise, loud noise, firearm noise, hypertension, and diabetes.

Table 7. OR for hearing loss by joint effect between blood lead and cadmium levels with the high-frequency method

\begin{tabular}{llll}
\hline Variable & Low lead & High lead & $\begin{array}{l}\text { Lead within strata of } \\
\text { cadmium }\end{array}$ \\
\hline Low cadmium & Ref. & $1.71(1.17-2.50)$ & $\begin{array}{l}1.71(1.17-2.50) \\
\text { High cadmium }\end{array}$ \\
Cadmium within strata of lead & $1.83(1.20-2.77)$ & $1.72(1.16-2.54)$ & $0.94(0.66-1.39)$ \\
\hline
\end{tabular}

Values in parentheses are 95\% CI. Hearing loss was defined as PTA $>25 \mathrm{~dB}$. ${ }^{\mathrm{b}}$ High-frequency method: PTA at 3, 4, and $6 \mathrm{kHz}$. Measurement of interaction on AN additive scale: RERI $=-0.82(-1.74$ to 0.09$) ; p=0.078$. Measurement of interaction on A multiplicative scale: ratio of $\mathrm{OR}=0.55$ (0.33 to 0.92); $p=0.023$. Models were adjusted for age, sex, monthly income, education level, smoking status, BMI, occupational noise, loud noise, firearm noise, hypertension, and diabetes. 
exposures), was 1.72 (95\% CI 1.16, 2.54). The OR was 1.71 (95\% CI 1.17-2.50) for participants with a high lead exposure only, and 1.83 (95\% CI 1.20-2.77) for participants with a high cadmium exposure only (Table 7). The estimate of the joint effect on the additive scale of blood lead and cadmium levels, the relative excess risk due to interaction, was -0.82 (95\% CI -1.74 to 0.09$)$. The observed effect was smaller than the sum of the estimated effects of blood lead and cadmium levels alone. Therefore, a negative interaction on the additive scale was observed, and the result approached statistical significance $(p=0.078)$. The ratio of OR, i.e., the estimate of the joint effect on a multiplicative scale, was 0.55 (95\% CI 0.33-0.92). The observed OR was smaller than the product of the estimated effects of blood lead and cadmium levels alone. Therefore, there was a negative interaction on the multiplicative scale, and the result was significant $(p=0.023)$. In the low-frequency and speech frequency methods, however, the measurements of interaction on both additive and multiplicative scales were not statistically significant (Tables 5,6$)$.

\section{Discussion}

This study illustrates the degree of hearing loss caused by environmental exposure to lead and cadmium, calculated using different PTA methods, in the participants of the KNHANES 2010-2012. The age-adjusted geometric means of blood lead and cadmium levels in the whole population were 2.46 and $1.02 \mu \mathrm{g} / \mathrm{L}$, respectively. It did not exceed the reference levels designated by the Centers for Disease Control and Prevention [2015] (blood lead level $<5 \mu \mathrm{g} / \mathrm{dL})$ and the World Health Organization [2010] (blood cadmium level $<5 \mu \mathrm{g} / \mathrm{L}$ ). We observed degeneration of the hearing ability of both the ears, at $4 \mathrm{kHz}$, with increasing blood lead and cadmium levels. There were no significant associations between hearing loss and blood levels of lead and cadmium with use of the lowfrequency $(0.5,1$, and $2 \mathrm{kHz})$ and speech frequency methods $(0.5,1,2$, and $4 \mathrm{kHz})$. In the high-frequency PTA method $(3,4$, and $6 \mathrm{kHz})$, however, blood lead and cadmium levels were significantly associated with hearing loss. In addition, we observed a negative interaction between lead and cadmium on hearing loss, only when the high-frequency PTA method was used.

A few previously conducted studies have reported the association between hearing loss and environmental exposure to heavy metals. In a recent study of 3,389 adolescents aged 12-19 years, in the US NHANES, the OR for hearing loss of the highest quartile of urinary cadmium was 3.08 (95\% CI 1.02-9.25) compared to the lowest urinary cadmium quartile in the case of PTA estimation using the frequencies $0.5,1$, and $2 \mathrm{kHz}$ [Shargorodsky et al., 2011]. Similarly, Choi et al. [2012] conducted a study of the general population in the USA and found that the OR for hearing loss of the highest quintile of blood cadmium was 1.74 (95\% CI 1.12-2.70), compared with the lowest blood cadmium quintile, when the PTA was calculated using the frequencies $0.5,1,2$, and $4 \mathrm{kHz}$. More studies have concentrated on lead exposure than on cadmium exposure. Chuang et al. [2007] calculated the PTA using the frequencies $0.5,1$, and $2 \mathrm{kHz}$ in a group of industry workers and reported that the PTA of the right and left ears were increased by 6.14 and $7.99 \mathrm{~dB}$, respectively, with a 10 -fold increment of the blood lead level. In addition, a longitudinal study of 448 men found that an increase in tibia lead levels of $15 \mu \mathrm{g} / \mathrm{g}$ was associated with an OR of 1.19 (95\% CI 0.92-1.53) and an increase in patella lead levels of $21 \mu \mathrm{g} / \mathrm{g}$ was associated with an OR of 1.48 (95\% CI 1.14-1.91) for hearing loss measured in the speech frequency [Park et al., 2010]. In a recent study on the general Korean population, participants in the highest quintile of blood lead (2.920-26.507 $\mu \mathrm{g} / \mathrm{dL})$ had an OR of 1.52 (95\% CI 1.11-2.10) for hearing loss in the frequencies 2, 3 , and $4 \mathrm{kHz}$, compared to those in the lowest quintile $(0.260-1.365 \mu \mathrm{g} / \mathrm{dL})$, with a significant linear trend [Huh et al., 2016].

Most studies pertaining to hearing loss caused by environmental exposure to heavy metals calculated the PTA using a relatively low-frequency range of $0.5-4 \mathrm{kHz}$. However, these studies may have underestimated the actual hearing loss caused by lead and cadmium exposure. When we examined the damage to hearing in each frequency, we found that impairment in hearing ability resulting from lead and cadmium exposure occurred more frequently in the high-frequency ranges than in the lowfrequency ranges. In our results, the $4-\mathrm{kHz}$ hearing thresholds increased with blood lead and cadmium levels, and the hearing loss at $6 \mathrm{kHz}$ in both the ears tended to increase with increasing blood lead concentrations, though there was no statistical significance. At $0.5,1$, and $2 \mathrm{kHz}$, however, the dose-response relationship between hearing loss and blood levels of lead and cadmium could not be evaluated. These results have been repeatedly reported in several epidemiological and toxicological studies [Hasan and Ozcaglar, 2009; Hwang et al., 2009; Park et al., 2010; Xue-wen et al., 2011; Choi and Kim, 2014; Liu et al., 2014], and they prove our hypothesis that hearing loss can be underestimated when the hearing loss caused 
by lead and cadmium exposure is evaluated using the PTA of low frequencies.

There are a few reasons behind the need to use highfrequency ranges to estimate hearing ability. First, it is rational to use higher frequencies because the more basal portion of the cochlea is more sensitive to pathology in general (particularly ototoxic chemicals but also noise and age-related effects). Second, we can identify not only hearing ability but also speech discrimination using highfrequency PTA. Someone with high-frequency hearing loss will be able to hear sounds but will have difficulty hearing sounds associated with the letters $\mathrm{F}, \mathrm{K}$, and $\mathrm{S}$ concentrated in a high-frequency range. The third reason is the social aspect. In field studies on the industries of Korea, Japan, and Taiwan, the presence of a hearing disorder was estimated using a sexenary method [Chuang et al., 2007], which weighted 1 - and $2-\mathrm{kHz}$ frequencies twice as much as $0.5-$ and $4-\mathrm{kHz}$ frequencies, $(0.5-\mathrm{kHz}$ threshold $+[1-\mathrm{kHz}$ threshold $\times 2]+[2-\mathrm{kHz}$ threshold $\times 2]+4-\mathrm{kHz}$ threshold)/6. However, because the weighting was done on relatively low-frequency hearing thresholds, this method can underestimate the hearing loss caused by lead and cadmium exposure more than the speech frequency PTA method. Thus, workers who have high-frequency hearing loss due to heavy metal exposure in the workplace have difficulty being admitted as victims of industrial accidents. For this reason, in Korea, people with hearing impairments have issued a statement requesting that high frequencies be taken into consideration when hearing loss is judged [Ablenews, 2009].

Another meaningful finding of this study is that a significant interaction of lead and cadmium on hearing loss was observed only when high-frequency PTA were used. Prior to this study, there was only one study that focused on the interaction of heavy metals on hearing loss. Choi et al. [2012] identified a positive interaction of the combined exposure to lead and cadmium in adults from the USA by calculating the PTA at $0.5,1,2$, and $4 \mathrm{kHz}$; however, no significance was found on either the additive or the multiplicative scale. In our study, the joint effect of lead and cadmium exposure on hearing loss was not significant when we used the low-frequency and speech frequency PTA (Tables 5, 6); this was the same as in the previous study. When we used the high-frequency PTA, however, a significant interaction was observed (Table 7). These results also suggest that low-frequency and speechfrequency PTA may not accurately reflect the hearing loss caused by lead and cadmium exposure.

Interestingly, the direction of interaction effects of exposure to lead and cadmium on hearing loss is negative; this is different from the results of previous studies. We illustrate this outcome in terms of 2 aspects: mechanism and concentration. Some parts of damaging mechanisms of lead and cadmium have an effect on the same position of the auditory organ. Previous studies on the mechanism of hearing loss through exposure to lead and cadmium have suggested that lead exposure can damage spiral ganglion neurons [Yamamura et al., 1989; Xue-wen et al., 2011] and cadmium exposure can damage hair cells, nerve fibers, and spiral ganglion neurons [Liu et al., 2014]. Therefore, the hearing damage caused by exposure to lead and cadmium on nerve cells may overlap when the exposures cooccur. Because of this overlap, the direction of the interaction effects may depend on the concentrations of lead and cadmium. If the exposure levels of heavy metals are not high enough to destroy the cells entirely, the joint effect on hearing loss may be greater than the sum of the individual effects because other damages are inflicted on the vulnerable cells due to an earlier attack. However, if the exposure levels of heavy metals are high enough to destroy the cells entirely, the joint effect on hearing loss may be smaller than the sum of the individual effects because the other attack is overlapped on already dead cells. According to this hypothesis, a positive interaction can be observed in cases of relatively low-level exposures and a negative interaction can be observed in cases of relatively high-level exposures. This topic will be discussed in detail in our future studies.

Our study has several strengths. This study used data from the KNHANES, which represents the general Korean population, and the results of our study can be generalized. We adjusted for potential confounders including noise exposure (i.e., occupational noise, recreational noise, and firearm noise), clinical risk factors (i.e., BMI, diabetes, and hypertension), and social economic status (family income and education level) better than previous studies. However, the limitations of this study should be considered. First, blood lead and cadmium levels may not be appropriate indicators of their cumulative exposure. Blood lead levels mainly reflect recent exposure [Hu et al., 2007]; a previous study used bone lead levels to identify an association between cumulative lead exposure and hearing loss [Park et al., 2010]. Blood cadmium levels generally reflect a recent exposure, whereas urinary cadmium points to a cumulative exposure. However, we used blood lead and cadmium levels instead of bone lead levels and urinary cadmium levels because the data from the KNHANES do not provide indicators of cumulative lead and cadmium exposure. In the case of low-level environmental exposure, both blood and urinary cadmium are 
considered to constitute the accumulated body burden in general population [Järup et al., 1998]. Second, we did not consider some potential confounders such as use of ototoxic medication (e.g., aminoglycoside antibiotics, chemotherapy agents, and loop diuretics) and consumption of selenium. The effect of medications confounds the effects of the toxins, and some participants would have had higher hearing thresholds due to medications regardless of their lead and/or cadmium exposure. In contrast, selenium has positive effects on the ears [Chuang et al., 2007], and therefore could have a protective effect on people with high selenium levels. Follow-up studies on hearing loss should consider the effects of selenium. Third, we cannot exclude the possibility of residual confounding because the variables pertaining to noise exposure were categorized as dichotomous variables. Finally, our study is a cross-sectional study, so we cannot be sure of the causal inference between exposure and hearing loss in our results.

\section{Conclusions}

This study provides evidence that the estimation of hearing loss is affected by the frequencies used in the PTA calculation. The individual and joint effects of lead and cadmium exposure on hearing loss were observed only when the high-frequency PTA method was used. These outcomes suggest that the high-frequency PTA method reflects hearing impairments caused by environmental exposure to lead and cadmium better than the low-frequency and speech frequency PTA methods.

\section{Statement of Ethics}

The KNHANES obtained written informed consent from all of the participants prior to conducting the survey, and the Institutional Review Board of the Korean Centers for Disease Control and Prevention approved this study (IRB No. 2010-02CON-21-C, 2011-02CON-06-C, 2012-01EXP-01-2C). We used secondary data from this survey for the epidemiology study.

\section{Disclosure Statement}

The authors declare that no conflict of interests exists.

\section{Funding Sources}

No sponsorship or funding was received for this study.

\section{References}

Ablenews: Korea: requiring of hearing impairment criteria to be reviewed. 2009. http://abnews.kr/q3F (accessed August 29, 2017).

Agrawal Y, Platz EA, Niparko JK: Prevalence of hearing loss and differences by demographic characteristics among US adults: data from the National Health and Nutrition Examination Survey, 1999-2004. Arch Intern Med 2008;168:1522-1530.

Centers for Disease Control and Prevention: Reference blood lead levels (BLLs) for adults in the US. 2015. http://www.cdc.gov/niosh/topics/ables/ReferenceBloodLevelsforAdults. html (accessed June 1, 2018).

Choi YH, Hu H, Mukherjee B, Miller J, Park SK: Environmental cadmium and lead exposures and hearing loss in US adults: the National Health and Nutrition Examination Survey, 1999 to 2004. Environ Health Perspect 2012; 120:1544-1550.

Choi YH, Kim K: Noise-induced hearing loss in Korean workers: co-exposure to organic solvents and heavy metals in nationwide industries. PLoS One 2014;9:e97538.
Choi YH, Park SK: Environmental exposures to lead, mercury, and cadmium and hearing loss in adults and adolescents: KNHANES 20102012. Environ Health Perspect 2017;125: 67003.

Chuang HY, Kuo CH, Chiu YW, Ho CK, Chen CJ, Wu TN: A case-control study on the relationship of hearing function and blood concentrations of lead, manganese, arsenic, and selenium. Sci Total Environ 2007;387:79-85.

Counter SA, Vahter M, Laurell G, Buchanan LH, Ortega F, Skerfving S: High lead exposure and auditory sensory-neural function in Andean children. Environ Health Perspect 1997;105: 522.

Hasan U, Ozcaglar BA: Effects of cadmium on the hearing system. Acta Otolaryngol 2009;121: 393-397.

Hosmer DW, Lemeshow S: Confidence interval estimation of interaction. Epidemiology 1992; 3:452-456.

Hu H, Shih R, Rothenberg S, Schwartz BS: The epidemiology of lead toxicity in adults: measuring dose and consideration of other methodologic issues. Environ Health Perspect 2007;115:455-462.
Huh DA, Choi YH, Moon KW: The effects of earphone use and environmental lead exposure on hearing loss in the Korean population: data analysis of the Korea National Health and $\mathrm{Nu}$ trition Examination Survey (KNHANES), 2010-2013. PLoS One 2016;11:e0168718.

Hwang YH, Chiang HY, Yen-Jean MC, Wang JD: The association between low levels of lead in blood and occupational noise-induced hearing loss in steel workers. Sci Total Environ 2009;408:43-49.

Järup L, Berglund M, Elinder CG, Nordberg G, Vanter M: Health effects of cadmium exposure - a review of the literature and a risk estimate. Scand J Work Environ Health 1998; 24:1-51.

Jones LG, Prins J, Park S, Walton JP, Luebke AE, Lurie DI: Lead exposure during development results in increased neurofilament phosphorylation, neuritic beading, and temporal processing deficits within the murine auditory brainstem. J Comp Neurol 2008;506:10031017. 
Kim S-J, Jeong H-J, Myung N-Y, Kim M-C, Lee J-H, So H-S, Park R-K, Kim H-M, Um J-Y, Hong S-H: The protective mechanism of antioxidants in cadmium-induced ototoxicity in vitro and in vivo. Environ Health Perspect 2008; 116:854.

Knol MJ, VanderWeele TJ: Recommendations for presenting analyses of effect modification and interaction. Int J Epidemiol 2012;41:514-520.

Korean Centers for Disease Control and Prevention: KNHANES V (2010-2012) Analysis Guideline (Guidebook for Data Users). Cheonju, KCDC, 2014.

Liu H, Ding D, Sun H, Jiang H, Wu X, Roth JA, Salvi R: Cadmium-induced ototoxicity in rat cochlear organotypic cultures. Neurotox Res 2014;26:179-189.
Musiek F: The Auditory System: Anatomy, Physiology, and Clinical Correlates. Boston, Pearson, 2006.

Park SK, Elmarsafawy S, Mukherjee B, Spiro A 3rd, Vokonas PS, Nie H, Weisskopf MG, Schwartz J, Hu H: Cumulative lead exposure and age-related hearing loss: the VA Normative Aging Study. Hear Res 2010;269:48-55.

Saunders JE, Jastrzembski BG, Buckey JC, Enriquez D, MacKenzie TA, Karagas MR: Hearing loss and heavy metal toxicity in a Nicaraguan mining community: audiological results and case reports. Audiol Neurootol 2013;18: 101-113.
Shargorodsky J, Curhan SG, Henderson E, Eavey R, Curhan GC: Heavy metals exposure and hearing loss in US adolescents. Arch Otolaryngol -Head Neck Surg 2011;137:1183-1189.

World Health Organization: Report of the Informal Working Group on Prevention of Deafness and Hearing Impairment Programme Planning. Geneva, WHO, 1991.

World Health Organization: Exposure to cadmium: a major public health concern. Geneva, WHO, 2010

Xue-wen W, Da-lian D, Hong S, Hong L, Hai-yan J, Salvi R: Lead neurotoxicity in rat cochlear organotypic cultures. J Otol 2011;6:43-50.

Yamamura K, Terayama K, Yamamoto N, Kohyama A, Kishi R: Effects of acute lead acetate exposure on adult guinea pigs: electrophysiological study of the inner ear. Fundam Appl Toxicol 1989;13:509-515. 